\title{
Study on the Dynamic Performance of a Modified Tuned Liquid Column Damper
}

\author{
M.F. Younes \\ Assistant professor, Mechanical Design Department, Faculty of Engineering, El-Mataria, \\ Helwan University, Cairo, Egypt , mf.fahmy@gmail.com
}

\begin{abstract}
The main objective of the present study is to reduce unwanted vibrations of mechanical systems through a sealed tuned liquid column damper (STLCD). A nonlinear mathematical model including nonlinear damping and air stiffness of the STLCD is considered to study the dynamic behaviour of a structure- STLCD coupled_system. A parametric study to investigate the influence of the main parameters of the STLCD such as length ratio and mass ratio on the dynamic behaviour of the main system subjected to ground excitation is presented. The air volume at rest is considered as an additional design parameter. The study showed that the efficiency and the range of application of the STLCD to control the system resonance vibration are better than the traditional one. In addition, it has been noticed that the gain in vibration mitigation from the STLCD with a bigger mass higher than $7 \%$ ratio is generally modest. Also the same trend is observed when increasing its horizontal length ratio over 0.8 . These enhance the actual implementation of the STLCD for small horizontal space applications. Equations for the optimum value of the initial air column height ratio and its corresponding performance index for the main structure-STLCD system as a function of both mass ratio and relative length were proposed.
\end{abstract}

Keywords: vibration control, passive control, tuned liquid damper, dynamic absorber, tuned liquid column damper 


\section{Introduction}

Modern engineering structures such as tall buildings, offshore platforms and wind turbines, etc., and new construction techniques have caused a raised need to construct relatively light and flexible units. Furthermore, many of those structures are usually subjected to dynamic forces, such as earthquakes, strong winds, huge waves, etc. which often cause a relatively large and serious vibrations leading to fatigue damage and poor safety. Therefore, many new ideas were developed to control the vibrations of these systems to a safe level. The installation of vibration absorbers on flexible structures are appropriate solutions for lessening the effects of serious vibrations [1-3]. In recent years, the tuned liquid column vibration absorber (TLCVA or TLCD) has been considered as effective in reducing system vibrations. The TLCVA consists of a rigid piping system integrated with the main system and partially filled with liquid, preferably water. The vibration attenuating capability is based on an energy transfer from the main system to the TLCVA, and which causes a relative motion of the liquid column. Consequently, the viscous and turbulent liquid damping, can be adjusted by the insertion of a hydraulic resistance (orifice plate), to dissipate the energy [4]. A previous research work has shown that TLCVA are very competitive to the traditional mass-spring-dashpot dynamic absorber to attenuate the vibration of wind turbine blades [5, 6]. In addition, a modified bidirectional TLCD that needs less liquid than the traditional TLCD was presented [7]. The experimental investigations show that the numerical proceedings are effective tools to evaluate the dynamic characteristics of the main system impeded with TLCD $[8,9]$. Several designs have been developed to improve the performance of the TLCD such as the pendulum type LCD, ball type LCD [10], compliant liquid column damper (CLCD) [11], adaptive spring -TLCD [12] and torsional TLCD [13].

Lately in another approach, a sealed tuned liquid column damper, STLCD was proposed as a new design of TLCVA. Where the ends of its vertical tubes are closed. This modification provides flexibility for tuning the frequency of STLCD through adjusting the initial air volume and pressure in addition to its geometrical shape [14, 15]. Reiterer [16] utilized the air spring effects in a STLCD to extend the applicability of the TLCD, which provides a reduction of the structural vibrations. Hochrainer [17] introduced simple guidelines for the optimal placement and the tuning of the STLCD. A compound STLCD to extend the range of the STLCD applications was proposed. [18]. Shum et al. [19] performed a theoretical analysis of the multiple STLCD to decrease the vibration of cable-stayed bridges under wind action. Moreover, the effectiveness of STLCD to control the vibration of the offshore platforms under seismic loading was studied [20, 21]. In addition, the performance of the STLCD to attenuate the torsional vibration of asymmetric structures was studied [22]. Bhattacharyya et al. [23] performed an experimental study on to the influence of the air spring nonlinearity on to the air pressure-volume relationship inside the STLCD.

Thereby, the total length and the cross-sectional area ratio are the only two parameters, which can be used for tuning the traditional TLCVAs. These limits the 
use of these types of absorbers to the applications having high weight and low frequency $(1 \mathrm{~Hz})$. On the other side, the STLCD, in addition to previous parameters, it can be tuned by regulating the air volume and pressure (air spring) in the vertical columns. The objectives of the current study are to attenuate the resonance response of the engineering structures having a natural frequency over $1 \mathrm{~Hz}$ by means of a STLCD, and to perform a parametric study of the main parameters of the structureSTLCD coupled system this includes the mass ratio and length ratio and structural damping. In addition, the influences of the air volume on the tuning and the efficiency of the STLCD with different geometrical configurations are also investigated. The nonlinear equations of motion (EOM) are derived and numerically solved. The optimum value of the air column height ratio and the performance index of the STLCD are obtained numerically through the minimization of the resonance response of the main system.

\section{Modelling of the STLCD}

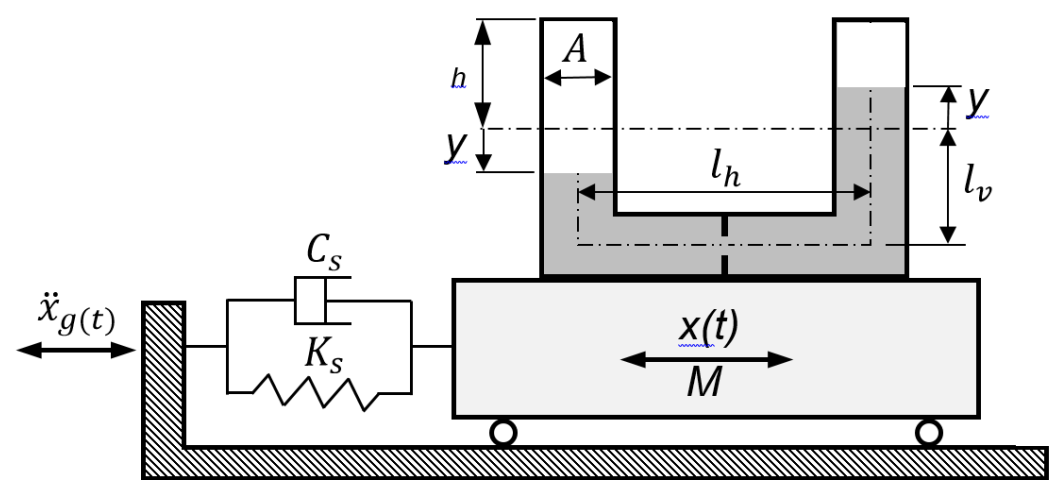

Fig. 1. Schematic diagram of structure-STLCD system.

\subsection{Modelling of air spring stiffness}

Referring to Fig. 1, The STLCD is a U-shaped pipe filled with a liquid. The lengths of the horizontal, vertical tube are $L_{h}$ and $L_{v}$ respectively and the total length of the liquid pipes is $L$. The two vertical columns are sealed at the ends with air of initial pressure $P_{o}$ and $V_{o}$ being the initial volume. When the STLCD to be subjected to an external excitation due to structural vibration. The air volume in the two vertical columns varies due to liquid motion inside the STLCD. The pressure-volume (P-V) relationship is expressed by the general polytropic relation as,

$$
P V^{n}=P_{o} V_{o}^{n}=\text { constant }
$$

Where $\mathrm{y}$ is the displacement of the liquid. The volume change $V$ and the net pressure between the two air columns $P$ can be described as:

$$
V=V_{o}-A y \text { and } P=P_{o} V_{o}^{n}\left(1-\frac{A y}{V_{o}}\right)^{-n}
$$


Where $n$ is the polytropic index ( $n=1: 1.4$ for air) [13-21], $A$ is the cross-sectional area of the STCLD and $h$ is the air column height. Therefore, the stiffness of the air spring in each vertical column may be obtained as:

$$
K=A\left(\frac{d P}{d y}\right)
$$

Substituting from Eq. (2) into Eq. (3) then,

$$
\begin{aligned}
K & =A P_{o} V_{o}^{n} \frac{d\left(1-A y / V_{o}\right)^{-n}}{d y} \\
& =\frac{n A^{2} P_{o}}{V_{o}}\left(1-A y / V_{o}\right)^{-(n+1)} \\
& =\frac{n V_{o} P_{o}}{h^{2}}(1-y / h)^{-(n+1)}
\end{aligned}
$$

\subsection{Equation of motion of STLCD}

If the liquid is incompressible, the velocity profiles of the liquid are uniform over the cross-sectional area of the STLCD and the coefficient of head loss is $\delta$ then, the equation of motion of the liquid column in the STLCD may be written as:

$$
\rho A L \ddot{y}+\frac{1}{2} \rho A \delta|\dot{y}| \dot{y}+2(\rho A g+K) y=-\rho A L_{h} \ddot{x}
$$

Where $\rho$ is the liquid density, $g$ is the gravity acceleration, and $\beta=y / h$ is the liquid displacement ratio. Therefore, the natural frequency of the STLCD $\omega_{a}$ can be expressed in the following form:

$$
\omega_{a}=\sqrt{2(\rho A g+K) / \rho A L}
$$

Substituting for $K$ in Eq. (6) from Eq. (4), we get:

$$
\omega_{a}=\sqrt{\frac{2 g}{L}\left(1+\frac{n P_{o}}{\rho g h}(1-\beta)^{-(n+1)}\right)}
$$

Equation (5) shows that the restoring and the damping force acting on the liquid inside the STLCD are nonlinear functions of the liquid displacement. Mostly, the numerical procedures are needed to solve Eq. (5) in the time domain. Equation (7) shows that the natural frequency of the STLCD is determined not only by the length of the liquid column but it can be increased by the factor $\left(\frac{n P_{o}}{\rho g h}(1-\beta)^{-(n+1)}\right)$ for a given liquid column length comparing with the traditional TLCD, which may facilitate the frequency tuning requirement.

\subsection{Equations of motion of structure - STLCD system}

Referring to Fig. 1, the structure - STLCD system is subjected to a ground acceleration $\ddot{x}_{g}(t)=(P G A) \sin (\omega t)$. The peak ground acceleration $P G A=g x f c$, $f_{c}, \omega$ are the intensity factor and frequency of the ground acceleration respectively. $M, C_{s}, K_{s}$ and $x(t)$ represent the mass, the equivalent viscous damping, stiffness and 
the displacement of the main system respectively, therefore, The EOMs for the structure-STLD coupled system can be expressed as:

$$
\begin{gathered}
\rho A L \ddot{y}+\rho A L_{h} \ddot{x}+\frac{1}{2} \rho A \delta|\dot{y}| \dot{y}+2(\rho A g+K) y=-\rho A L_{h} \ddot{x}_{g} \\
(M+\rho A L) \ddot{x}+\rho A L_{h} \ddot{y}+C_{s} \dot{x}+K_{s} x=-(M+\rho A L) \ddot{x}_{g}
\end{gathered}
$$

Normalization of Eq. (8) with respect to the mass of liquid $m(m=\rho A L)$ and main system mass $(M)$ respectively, leads to:

$$
\begin{gathered}
\ddot{y}+\alpha \ddot{x}+\frac{\delta}{2 L}|\dot{y}| \dot{y}+\omega_{a}^{2} y=-\alpha \ddot{x}_{g} \\
(1+\mu) \ddot{x}+\alpha \mu \ddot{y}+2 \zeta_{s} \omega_{n} \dot{x}+\omega_{n}^{2} x=-(1+\mu) \ddot{x}_{g}
\end{gathered}
$$

In which, $\omega_{n}=\sqrt{K_{s} / M}, \zeta_{s}=C_{s} / 2 M \omega_{n}, \alpha=L_{h} / L, \mu=m / M$ and $r=\omega / \omega_{n}$ are the natural frequency, damping ratio of the main system, length ratio ,mass ratio and frequency ratio respectively. Finally, the structure-STLCD equations of motion can be formulated in a matrix form as:

$$
\left[\begin{array}{cc}
(1+\mu) & \alpha \mu \\
\alpha & 1
\end{array}\right]\left\{\begin{array}{c}
\ddot{x} \\
\ddot{y}
\end{array}\right\}+\left[\begin{array}{cc}
2 \zeta_{s} \omega_{n} & 0 \\
0 & \frac{\delta}{2 L}|\dot{y}|
\end{array}\right]\left\{\begin{array}{c}
\dot{x} \\
\dot{y}
\end{array}\right\}+\left[\begin{array}{cc}
\omega_{n}^{2} & 0 \\
0 & \omega_{a}^{2}
\end{array}\right]\left\{\begin{array}{l}
x \\
y
\end{array}\right\}=-\left\{\begin{array}{c}
(1+\mu) \\
\alpha
\end{array}\right\} \ddot{x}_{g}
$$

The response of the structure-STLCD system in both the time and the frequency domains can be estimated by solving Eq. (10) using the Adaptive Runge-Kutta method.

\section{Parametric study and results}

Referring to Fig. 2, the time history results indicate that the presented nonlinear model provide identical solutions and in good agreement with the linear model results reported in the previous literature for small relative displacement [15]. The response ratio $x_{r}=\frac{x_{\max }}{x_{w}}$ is determined as the ratio of the peak displacement of the structure -STLCD system $\left(x_{\max }\right)$ to its peak displacement without a STLCD $\left(x_{w}\right)$. However, for high relative displacement when the linear model is not valid the presented nonlinear model is anticipated to give helpful results for a wide range of the liquid's displacement.
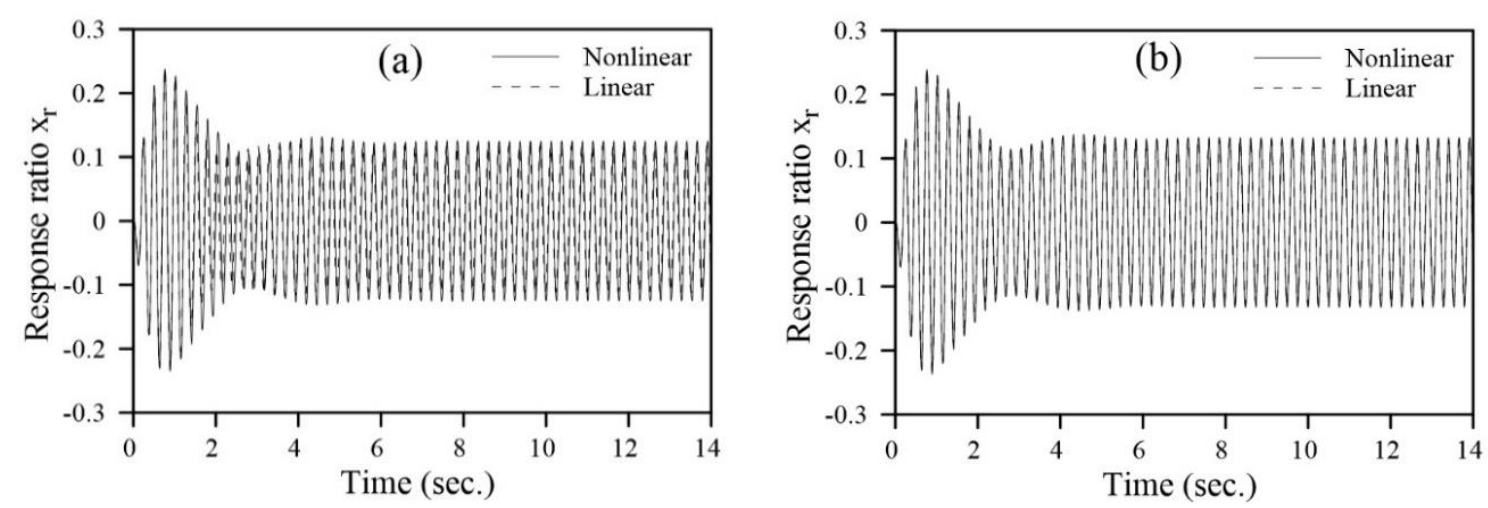

Fig.2. Response time histories of the structure-STLCD for $\mu=8 \%$ : a) $\alpha=0.5$, b) $\alpha=0.8$ 
Since the objective of installing the dynamic absorber to the structure is to bring the resonant peak down to its lowest possible value, some typical plots of frequency response curves under different values of the mass ratio are shown in Fig. 3 . Reffering to Fig. 3a it can be noticed that by increasing the mass ratio the minimum response ratio was decreased and shifted towards the right side, and one point from the two peaks of the curve is going up and the other is going down. Figure $3 \mathrm{~b}$ shows the influence of the mass ratio on relative liquid displacement $\varphi=y / L_{v}$ in the frequency domain. For excitation intensity and the length ratio equal to $0.1 \mathrm{~g}$ and 0.8 respectively. It is seen that for the whole values of the mass ratio the relative liquid displacement is acceptable (less than 0.5 ) to maintain the U-shape and continuity of the liquid column. In addition, the minimum liquid displacement takes place at resonance conditions and its value is smaller than the reported value in case of the traditional TLCD [3]. This may allow extending the using of the STLCD to the applications under strong excitation.

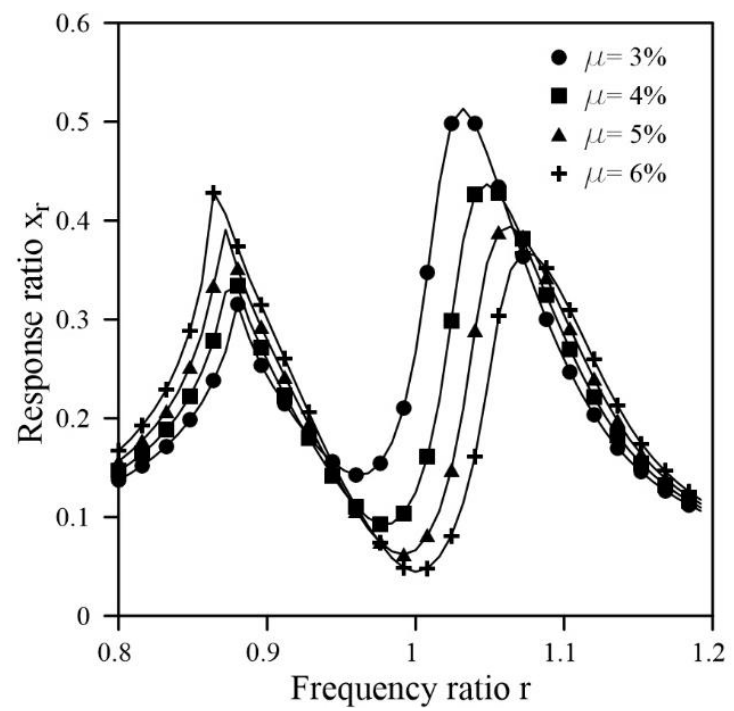

(a)

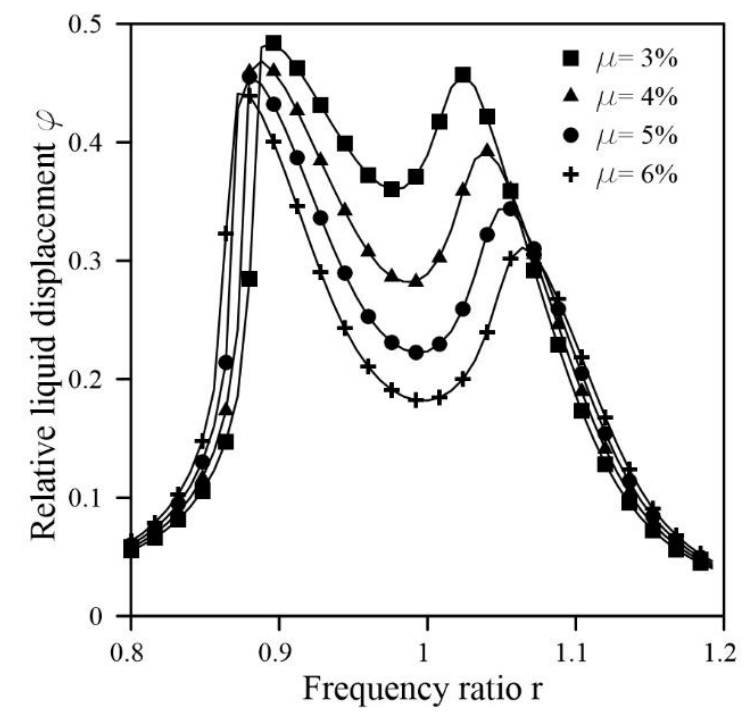

(b)

Fig. 3. Frequency response curve of the structure-STLCD system

Figure 4 shows the variation of the maximum $x_{\text {max }}$, mean $x_{\text {mean }}$ and minimum $x_{\text {min }}$ response ratios of the main system with the mass ratio respectively, in the excitation frequency ratio range $r=0.8$ to 1.1 , the frequency tuning ratio $r_{a}$ is unity and $\alpha=0.8$. It can be observed that the STLCD with a bigger mass can achieve lower minimum response ratio (better performance) with a slight change in mean response ratio. But on the another side, the maximum response ratio decreases with the mass ratio until it reaches to the minimum value at $\mu \cong 5 \%$ then it began to increase by increasing the mass ratio over $\mu=5 \%$, this due to the shifting of the two peaks as discussed previously. This behaviour may indicate that the minimum response ratio at $r_{a}=1$ may not take place at resonance state. On another side, the gain on the reduction on the system response was decreased by increasing the mass ratio over than $(\mu=5$ : $6 \%$ ) which also give the minimum value of $x_{\max }$. Referring to Fig. 4 , when the mass ratio increases from $5 \%$ to $6 \%$ the minimum response ratio decreases by about 
$30 \%$. While when $\mu$ increases from $7 \%$ to $8 \%$, there is a decrease in $x_{\min }$ by only about 21\%. Figure 5 shows the influence of the volume ratio $V_{r}\left(V_{r}=\right.$ intial air volume/liquid volume $)$ on the response ratio $x_{r}$ at resonance excitation for various values of $\mu$. The length ratio is equal to 0.8 and the liquid length is kept constant. As shown in Fig. 5, the effect of the STLCD to reduce the main system response has some dependency on the initial air volume, but this dependency is in an uneven way. The smallest response ratio at resonance excitation for mass ratios $4 \%$ and $6 \%$, occurred at about $V_{r}=11 \%$ and $11.6 \%$ respectively.

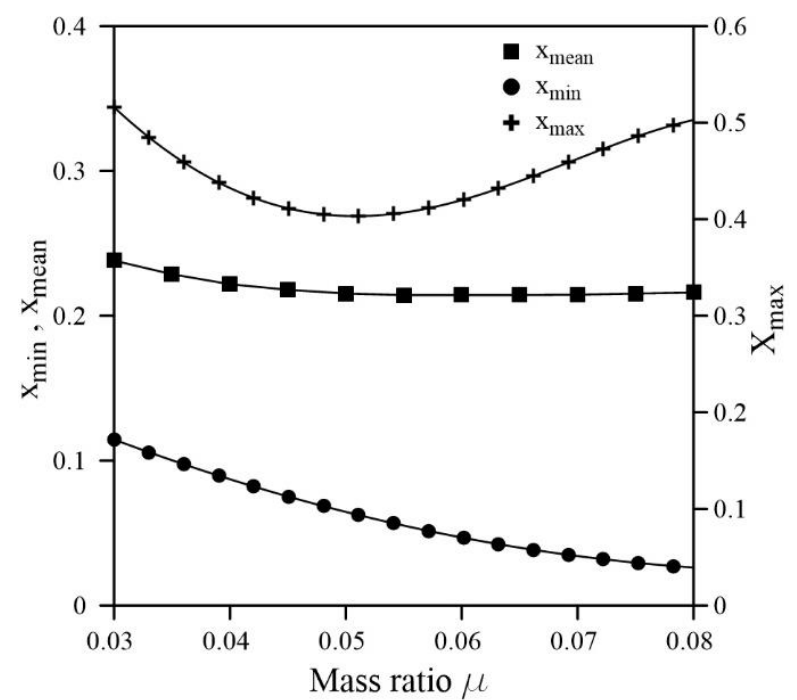

Fig. 4. The effect of mass ratio on system response $x_{\text {max }}, x_{\text {mean }}$ and $x_{\text {min }}$.

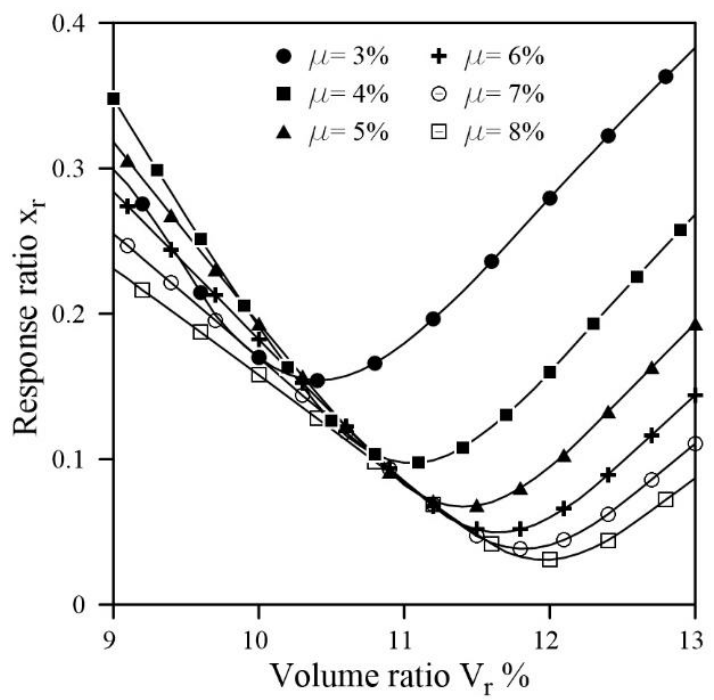

Fig. 5. Variation of $x_{r}$ with volume ratio for different mass ratios.

Figure 6 shows, The effect of the mass ratio on the response ratio when $V_{r}=$ constant, and $V_{r} \neq$ constant with the mass ratio in the range of 3 to $8 \%$. It can be seen that the volume ratio for maximum reduction in system response increases as the mass ratio is increased. The gains from the tuning of the volume ratio are about $30 \%$ and $40 \%$ for the mass ratio of $\mu=3 \%$ and $8 \%$ respectively. Figure 7 shows the variation of the performance index $\left(\eta=1-x_{r}\right)$ with the volume ratio for various values of the structural damping and mass ratio. It can be noticed that the effect of the structural damping on to the volume ratio of the maximum performance index is very insignificant. The performance index is slightly decreased as the main system damping is increased such as the structural damping is increased from $1 \%$ to $2 \%$ the performance index is reduced by about $2.4 \%$ and $1.1 \%$ for the mass ratio of $5 \%$ and $8 \%$ respectively. Inspecting Fig. 8 , it is apparent that the liquid displacements $\varphi$ and $\beta$ have been considered acceptable with the small effect of on their values by changing the structural damping. In addition, it can be seen that the liquid displacement ratio $\beta$ began to decreasing when volume ratio reached to its tuning value which corresponding to frequency tuning ratio $r_{a}=1.037$. With the constant total liquid length and the frequency of the main system equal to $4 \mathrm{~Hz}$, the variation of the performance index of the structure-STLCD system are shown in Fig. 9 for varying combination of volume 
ratio and the length ratio of the STLCD. The radar plots are shown for two different mass ratios $\mu=3 \%$ and 5\% in Fig. 9a and 9b. Similar plots for the two alternative mass ratios $\mu=7 \%$ and $8 \%$ are shown in Fig. $9 \mathrm{c}$ and $9 \mathrm{~d}$. It is observed that there are volume tuning ratios to attain the minimum system responses and thereby maximizing the control efficiency and the performance index. It is also noted that the optimum value of the tuning ratios and the corresponding performance index increase with increasing the mass ratio and the length ratio.

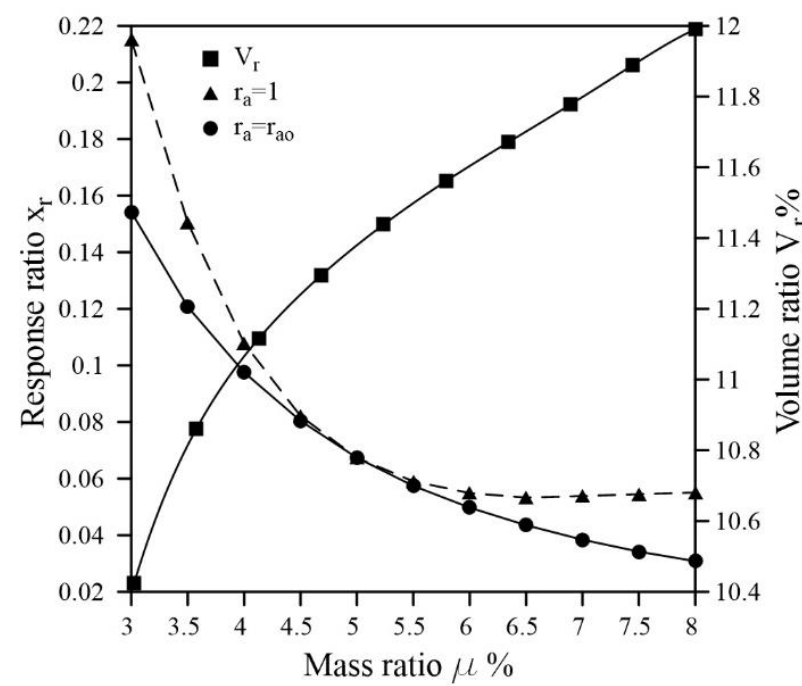

Fig. 6. The effect of mass ratio on $V_{r}$ and the corresponding response ratio

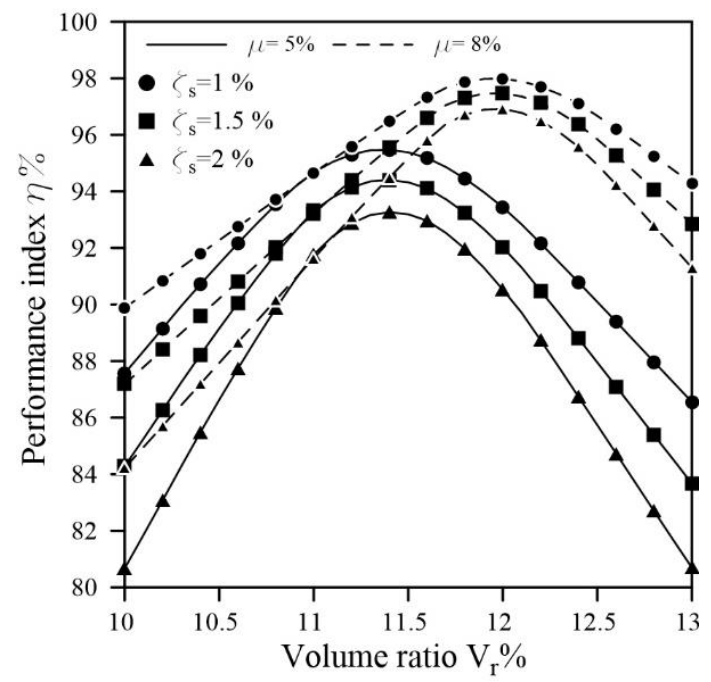

Fig.7. Variation of performance index with respect to $V_{r}$ and $\zeta_{s}$

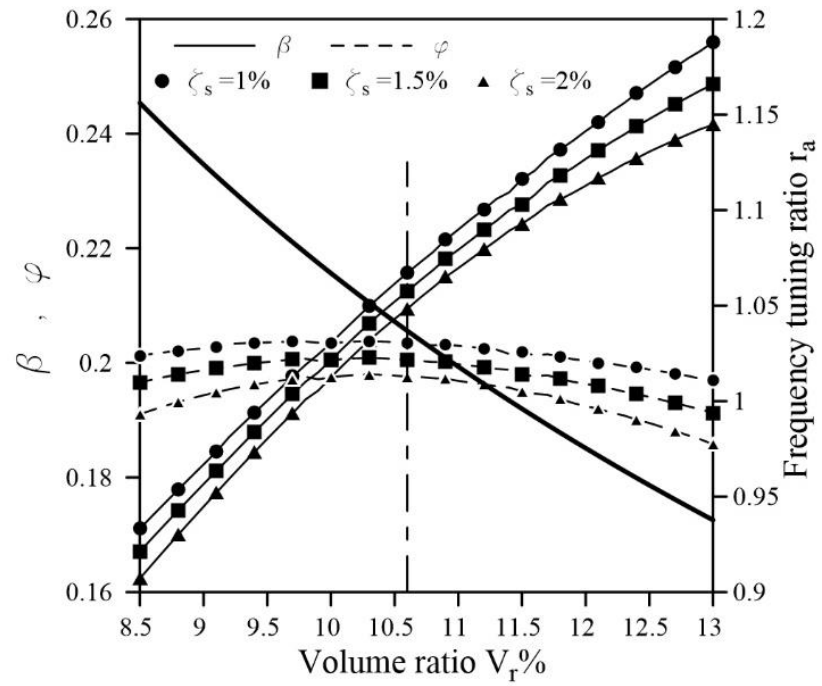

Fig.8. Liquid displacement ratio and tuning frequency ratio for different $V_{r}$ and $\zeta_{s}$.

Referring to Fig. 9, the performance index increases by about 9\%, 2.5\% and $0.8 \%$ with increasing the mass ratio from $3 \%$ to $5 \%$, from $5 \%$ to $7 \%$ and from $7 \%$ to $8 \%$ respectively. However, after a particular value, by increasing the mass ratio, the efficiency of the STLCD increases with a slow rate, and one can expect that after a threshold by raising the mass ratio, the efficiency of the STLCD would reduce. 
However, higher mass ratio of the STLCD results in heavy damper to change the overall dynamic property of the combined structure-damper system and the associated increase in cost. Therefore, the practical value of this mass moment ratio should be kept as small as possible, typically around 5\% for the STLCD. Referring to Fig. 9, an important feature to observe in STLCD is that the performance index remains acceptable irrespective of the length ratio, whereas, in traditional TLCD, the efficiencies much decrease with decreasing the length ratio. Where, the minimum performance index reported at $\mu=3 \%$ and $\alpha=0.5$ is $61 \%$ and it increases to $79 \%$ and $87 \%$ with increasing the mass ratio to $5 \%$ and $7 \%$ respectively. This implies to the enhanced performance robustness of the STLCD under varying length ratios. In addition, it can be noticed that there is a very little improvement in the performance index with increasing the length ratio from 0.8 to 0.9 by about $1.8 \%$ and $1 \%$ at $\mu$ equal to 5\% and 7\% respectively. A larger $\alpha$ will even result in a bigger demand in horizontal space, which is not possible to be achieved in actual applications.
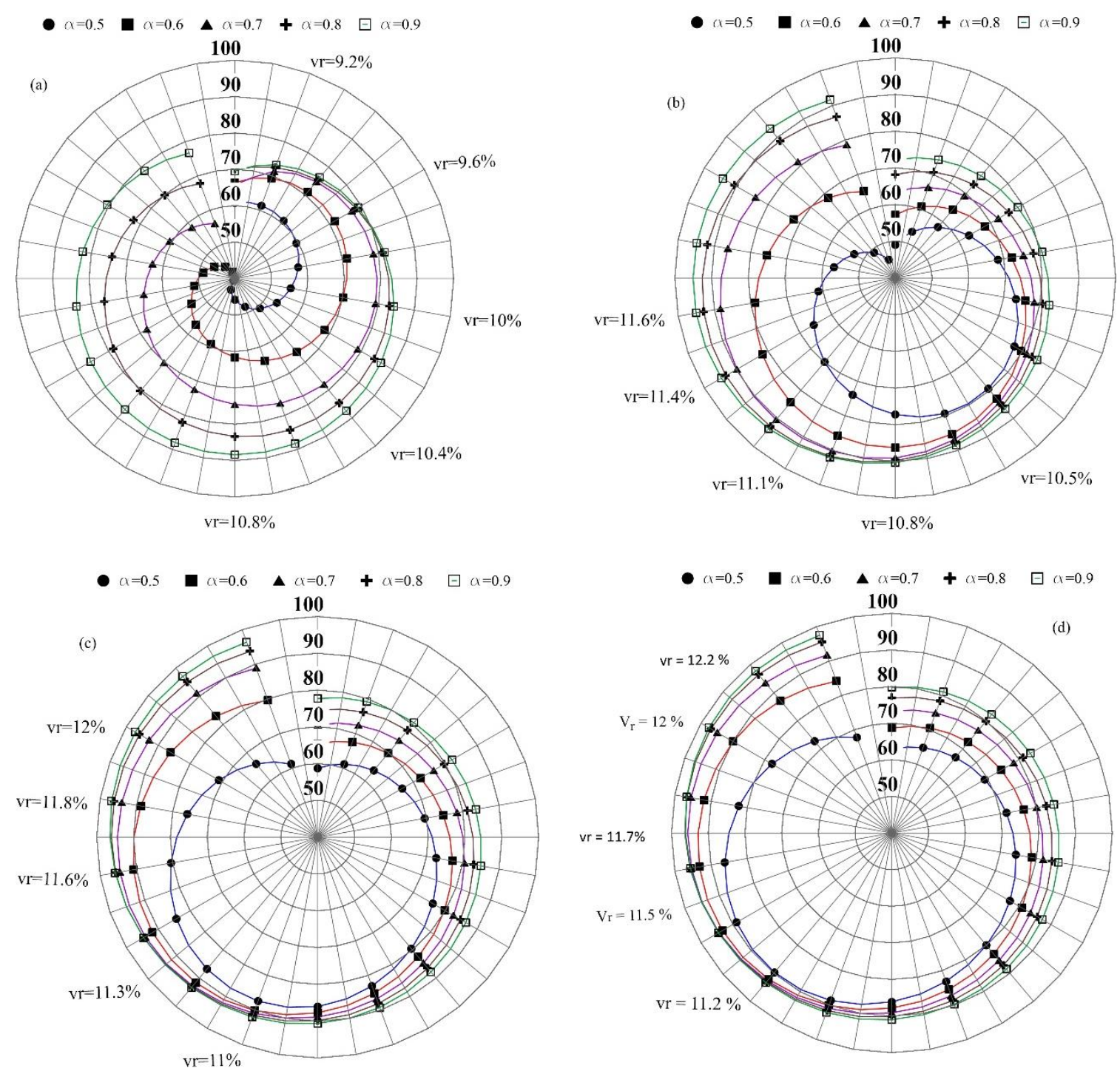

Fig. 9. Variation of performance index with volume ratio for different $\alpha$ and $\mu$ 


\section{Parametric optimization}

To optimize the STLCD parameters for achieving the best vibration attenuation of the structure a constrained optimization problem is formulated and solved numerically. The designer can specify the mass ratio and the length ratio according to space constraints. The air column height ratio $\gamma=h / L_{v}$ is the variable considered to evaluate its optimal values. The objective of the current optimization process is to minimize the resonance response ratio $x_{r}$. The constraint is applied by restricting the maximum liquid displacement ratio to half $\left(\beta_{\max } \leq 0.5\right)$.

The optimization problem is defined as:

Find $\quad X\left(\gamma_{o}\right)$

To minimize $\quad F(X)=x_{r}$

Subject to $G(X) \leq 0.5$ where $G(X)=\beta_{\max }$,

With $l b \leq \gamma_{o} \leq u b$

Where $\gamma_{o}$ represents the required optimal values for air column height ratio which corresponding to the optimal volume ratio where the resonance frequency response is minimal. $l b$ and $u b$ are the lower and upper bounds which can be expected from the parametric study.

Since the above defined constrained optimization problem and the variable is of a continuous type thus, it is a nonlinear complex problem. Global Search Algorithm (GSA) is implemented to obtain the optimal solution. The GSA is a numerical optimization technique, which utilizes the scatter search algorithm to produce a series of trial points as start points within limited boundaries and has an efficient nonlinearity constrained multivariable optimization solver. The GSA tests the start points and then takes only the points with a good probability of achieving a global minimum to run the solver from these points. The solver (fmincon) utilizes the sequential quadratic programming (SQP) technique to optimize the object function. The formularization of SQP is based on Newton's method and Karush-KuhnTucker (KKT) equations. KKT equations based algorithms try to get the Lagrange multipliers directly Eqs. (12-14).

$$
L(X, \lambda)=F(X)+\lambda \cdot G(X)
$$

At the $k^{\text {th }}$ iteration with $p\left(p_{1 k}, p_{2 k}\right)$, the following quadratic programming sub problem is solved:

Subject to

$$
\min _{p} \frac{1}{2} p^{T} \nabla_{x x}^{2} L_{x} p+\nabla F_{k}^{T} p+f_{k}
$$

Figure 10 shows general outline of the optimization problem formulation.

According to the obtained results and referring to Fig. 11, the air column height ratio depends on the mass ratio and the length ratio. Using OriginLab's surface fitting tools, the following relationships are proposed for the optimum air height 
ratio and its corresponding performance index. It should be noted that the PGA of ground acceleration is not presented in Eqs. $(15,16)$ because the head loss coefficient would reflect its effect.

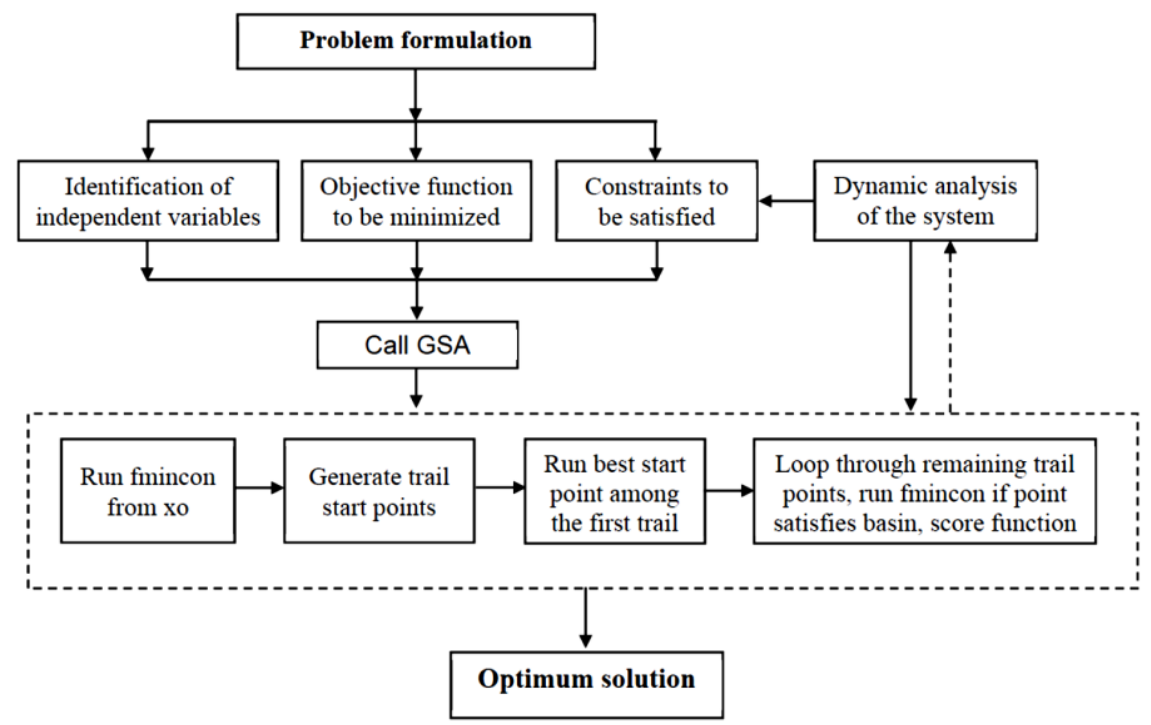

Fig.10. Optimization problem outline
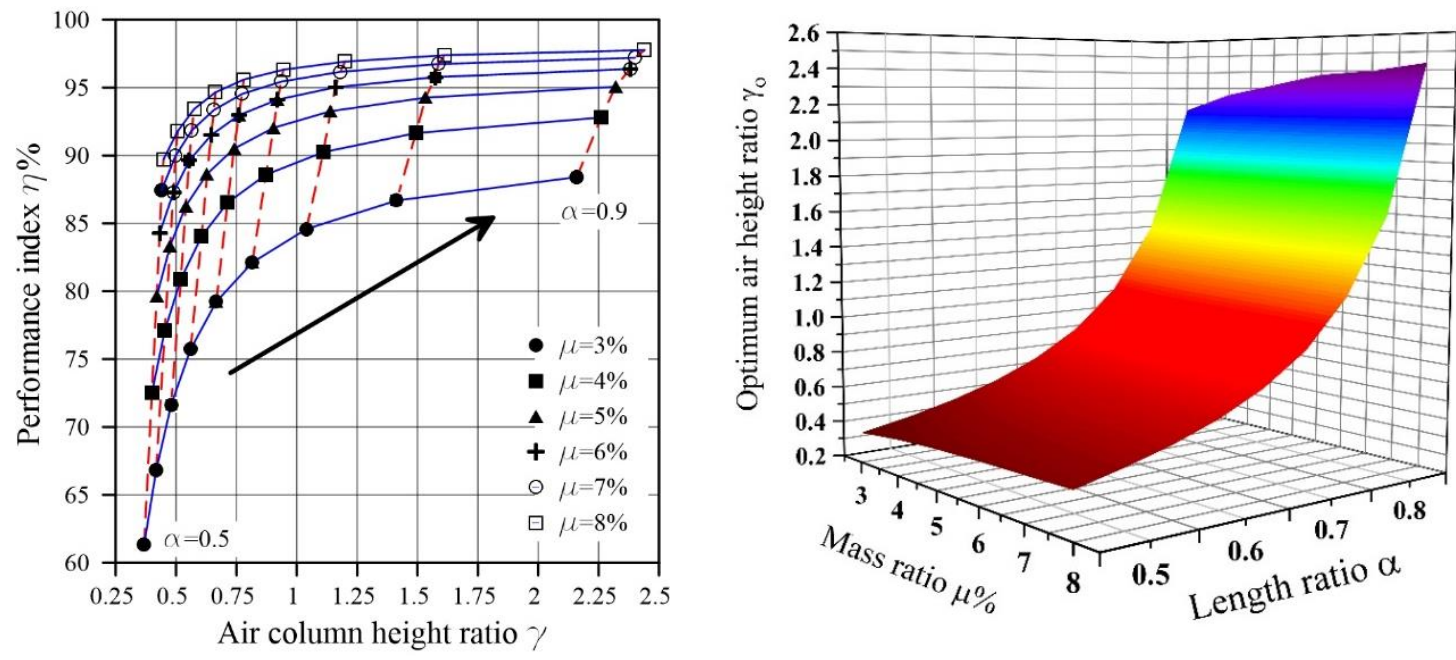

Fig. 11. Optimum air column height ratio and performance index for different mass and length ratios

$$
\gamma_{o}=-0.0816+\left(\frac{15.978}{1+A_{1}\left(1+A_{2}\right)}\right)+(0.8046)\left(\exp (-0.5)\left(A_{1}+A_{2}\right)\right)
$$

$\eta \%=\frac{-1.0475+876.6297 \mu-57.2323 \alpha+102.6262 \alpha^{2}+78.5827 \alpha^{3}}{1-8.594 \mu+236.5038 \mu^{2}-1129.34 \mu^{3}-2.3051 \alpha+3.0119 \alpha^{2}}$

Where,

$A_{1}=((\mu-0.08069) / 0.13463)^{2}$ and $A_{2}=((\alpha-1.36879) / 0.15911)^{2}$ 


\section{Conclusions}

The nonlinear equations of motion of the structure-STLCD coupled system including nonlinear damping and stiffness of the STLCD were solved numerically to perform a parametric study for the STLCD and its contributions on the dynamic performance of the structures have a natural frequency up to $4 \mathrm{~Hz}$. Optimum initial air volume in term of air column height ratio was evaluated for different configurations of mass ratio and the relative length. The results show that the air spring allows the extension of the practical frequency of the STLCD several times over the traditional one where its frequency is restricted by liquid length only. Furthermore, the greater the relative length and the mass ratio, the greater the efficiency of the STLCD due to the increase of the accelerated liquid in the horizontal portion. On the other side, a very little influence on the performance index was observed by raising the length ratio and the mass ratio over 0.8 and $7 \%$ respectively. The numerical optimization results show that the optimum air volume ratio increases when increasing the mass ratio and the horizontal length ratio and it is slightly affected by the main system damping. Its value was varied from $10.5 \%$ to $12 \%$ when increasing the mass ratio from $3 \%$ to $7 \%$ and horizontal length ratio from 0.5 to 0.9 with performance index about $61 \%$ and $97 \%$ respectively. This supports the use of the STLCD for the applications having a restricted horizontal space. The optimum values of the air column height ratio and the corresponding performance index can be obtained directly from the presented optimization equations, without any other iteration or modification, resulting in a very significant reduction in computational efforts. 


\section{References}

1. A.k. Mallik, S. Chatterjee, Principles of passive and active vibration control, affiliated east-west press private limited, New Delhi, 2014.

2. M.F. Younes, Optimal design of dynamic vibration absorber for rolling systems, Journal of engineering and applied science, vol. 64, pp. 307-324, 2017.

3. M. F. Younes, Numerical modelling and experimental investigation of a single and a compound pendulum impact dynamic vibration absorbers, $17^{\text {th }}$ international conference on aerospace sciences and aviation technology, April 11 - 13, pp. 1-14, 2017.

4. M. F. Younes, Effect of different design parameters on damping capacity of liquid column vibration absorber, Journal of engineering and applied science, vol. 65, no. 6, pp. 447-467, 2018.

5. Z. Zhang, H. Christian, Vibration control of floating offshore wind turbines using liquid column dampers, Journal of physics: conf. series 1037032002 , 2018.

6. B. Basu, Z. Zhang, S.K. Nielsen, Damping of edgewise vibration in wind turbine blades by means of circular liquid dampers, Journal of wind energy, vol. 19, pp. 213-226, 2016.

7. L. Rozas, R.L. Boroschek, A. Tamburrino, M. Rojas, A bidirectional tuned liquid column damper for reducing the seismic response of buildings, Journal of struct. control health monit., vol.23, pp. 621-640, 2016.

8. A.C. Altunisik, A. Yetisken, V. Kahya, Experimental study on control performance of tuned liquid column dampers considering different excitation directions, Journal of mech. syst. signal process, vol. 102, pp. 59-71, 2018.

9. A. Matteo, M. Paola, A. Pirrotta, Innovative modelling of tuned liquid column damper controlled structures, Journal of smart struct. syst., vol. 18, 117-138, 2016.

10. K.A. Al-saif, K.A. Aldakkan, M.A. Foda, Modified liquid column damper for vibration control of structures, Journal of mechanical sciences, vol. 53, pp. 505-512, 2011.

11. S. Bhattacharyya, A.D. Ghosh, B. Basu, Experimental investigations into CLCD with identification of tuning and damping effects, Journal of structural engineering, vol. 143,issue 9, 2017.

12. E. Sonmez, S. Nagarajaiah, C. Sun, B. Basu, A study on semi-active tuned liquid column dampers (stlcds) for structural response reduction under random excitations, Journal of sound and vibration, vol. 362, pp. 1-15, 2016.

13. M. Hochrainer, Control of vibrations of civil engineering structures with special emphasis on tall buildings, Doctoral dissertation, Vienna University of technology, Austria, 2001.

14. W.D. Corte, C. Delesie, Sealed tuned liquid column dampers: a cost effective solution for vibration damping of large arch hangers, $5^{\text {th }}$ 
international conference on arch bridges, Madeira, Portugal, pp. 409-416, 2007.

15. F. Ziegler, A. kazemiamiri, Bridge vibrations effectively damped by means of tuned liquid column gas dampers, Asian journal of civil engineering, vol. 14, pp. 1-16, 2013.

16. M. Reiterer, F. Ziegler, Bi-axial seismic activation of civil engineering structures equipped with tuned liquid column dampers, Journal of seismology and earthquake engineering, vol. 7, pp. 45-60, 2005.

17. M. Hochrainer, F. Ziegler, Control of tall building vibrations by sealed tuned liquid column dampers, Journal of structural control and health monitoring, vol. 13, pp. 980-1002, 2006.

18. M. Hochrainer, P. A. Fotiu, Design of coupled tuned liquid column gas dampers for multi-mode reduction in vibrating structures, Journal Acta Mechanica, vol. 229, pp. 911-928, 2018.

19. K.M. Shum, Y.L. Xu, W.H. Guo, Wind-induced vibration control of long span cable-stayed bridges using multiple pressurized tuned liquid column dampers, Journal of wind eng. ind. aerodyn., vol. 96, pp. 166-192, 2008.

20. S.A. Mousavi, K. Bargi, S.M. Zahrai, Optimum parameters of tuned liquid column-gas damper for mitigation of seismic-induced vibrations of offshore jacket platforms, Journal of structural control and health monitoring, vol. 20, pp. 422-444, 2013.

21. S.A. Mousavi, S.M. Zahrai, k. Bargi , Optimum geometry of tuned liquid column-gas damper for control of offshore jacket platform vibrations under seismic excitation, Journal of structural control and health monitoring, vol. 1, pp. 579-592, 2012.

22. F. Chuan, Application of torsional tuned liquid column gas damper for planasymmetric buildings, Structural Control and Health Monitoring, Vol.18, pp. 492-509, 2012.

23. S. Bhattacharyya, A. Ghosh, B. Basu, Nonlinear modelling and validation of air spring effects in a sealed tuned liquid column damper for structural control. Journal of sound and vibration, vol. 410, pp. 269-286, 2017. 